\title{
Clima organizacional y desempeño laboral, caso: empresa Lechera Peruana
}

\section{Organizational environment and work performance, case: Peruvian dairy company}

Doi: https://doi.org/10.22458/rna.v1 1 i2.3297

Ing. Néstor Humberto Chagray Ameri

Universidad Nacional José Faustino

Sánchez Carrión, Perú

nestorameri7@gmail.com

https://orcid.org/0000-0002-4962-8582

Ing. Rufino Máximo Maguiña Maza

Universidad Nacional José Faustino

Sánchez Carrión, Perú

rmaguina@unjfsc.edu.pe

https://orcid.org/0000-0001-7795-5727
Dr. Santiago Ernesto Ramos Y Yovera

Universidad Nacional José Faustino

Sánchez Carrión, Perú

sramos@unjfsc.edu.pe

https://orcid.org/0000-0003-3674-0302

Mg. Sc. Yaneline Nilda Hidalgo Vasquez

Universidad Nacional José Faustino

Sánchez Carrión, Perú

yanihiva@gmail.com

https://orcid.org/0000-0002-8258-5991

\author{
Dr. Abrahan Cesar Neri Ayala \\ Universidad Nacional José Faustino \\ Sánchez Carrión, Perú \\ aneri@unjfsc.edu.pe \\ https://orcid.org/0000-0003-2799-3244
}

Fecha de recepción: 10 de abril de 2020

Fecha de aceptación: 24 de agosto de 2020

\section{RESUMEN}

El ambiente existente entre los miembros de una empresa determina un clima organizacional, el cual se basa en estados emocionales como resultado de la percepción de los trabajadores sobre los diferentes aspectos dentro de su empresa. Por lo tanto, el clima organizacional se refleja en el comportamiento y desempeño de los trabajadores. Este estudio muestra las relaciones encontradas entre las dimensiones del clima organizacional y el desempeño laboral. Los resultados demuestran que el liderazgo y las relaciones interpersonales se correlacionan significativamente con el desempeño laboral.

\section{ABSTRACT}

The current environment among the members of a company determines an organizational environment based on emotional states as a result of the workers' perception of various aspects within their company. Therefore, the organizational environment is reflected in the workers' behavior and performance. This study shows the relationships found between the dimensions of organizational environment and work performance. The results demonstrate that leadership and interpersonal relationships are significantly correlated with work performance.

\section{RÉSUMÉ}

L'ambiance qui existe entre les membres d'une entreprise détermine un climat organisationnel qui est basé sur des états émotionnels comme résultat de la perception des travailleurs sur les différents aspects au sein de leur entreprise. Par conséquent, le climat organisationnel se reflète dans le comportement et rendement des travailleurs. Cette étude montre les relations identifiées entre les dimensions du climat organisationnel et le rendement professionnel. Les résultats démontrent que la capacité de diriger et les relations interpersonnelles sont en corrélation significative avec le rendement professionnel.

\section{RESUMO}

$\mathrm{O}$ ambiente entre os membros de uma empresa determina um clima organizacional, o qual se baseia nos estados emocionais decorrentes da percepção dos trabalhadores sobre os diferentes aspectos de sua empresa. Portanto, o clima organizacional se reflete no comportamento e desempenho dos trabalhadores. Este estudo mostra as relações encontradas entre as dimensões do clima organizacional e o desempenho no trabalho. Os resultados demonstram que a liderança e o relacionamento interpessoal estão significativamente correlacionados com o desempenho no trabalho.

\section{PALABRAS CLAVE:}

ADMINISTRACIÓN,

DESEMPEÑO,

ORGANIZACIÓN,

COLABORADORES
KEYWORDS:

ADMINISTRATION,

PERFORMANCE,

ORGANIZATION,

COLLABORATORS.
PALAVRAS CHAVE:

ADMINISTRAÇÃO,

DESEMPENHO,

ORGANIZAÇÃO,

COLABORADORES.
MOTS CLÉS:

ÉTHIQUE,

FINANCES,

PERTINENCE,

RESSOURCES,

SÉCURITÉ. 


\section{INTRODUCCIÓN}

La actividad ganadera en el Perú tiene gran importancia, ya que representa cerca del $40 \%$ del Valor Bruto de la Producción Agropecuaria, siendo fundamental en la seguridad alimentaria. Esta actividad se realiza en las regiones de la Costa, Sierra y Selva, bajo diferentes sistemas de producción, con base en los recursos disponibles de cada región, aplicando tecnologías, con la finalidad de lograr una ganadería competitiva (Minagri, 2017).

Es así que las empresas dedicadas a la producción de leche, al igual que las empresas de todo rubro, están en constante búsqueda de innovación y de un crecimiento tanto interno como externo para lograr ser competitivas. Por ello, el clima organizacional y el desempeño laboral son indicadores que se pueden analizar para encaminar la toma de decisiones, la resolución de conflictos y lograr el éxito de la organización.

La identificación y análisis del clima organizacional en las empresas lecheras permite el mejoramiento continuo del ambiente de trabajo. El tener conocimiento de aquellos factores que repercuten en el clima organizacional, permite la detección de las causas de los conflictos que generan una actitud negativa por parte del personal. Por ello, el análisis del clima organizacional, permite lograr la eficiencia y productividad de sus sistemas de producción, el desarrollo del talento humano y la calidad de vida del personal (Beltrán y Téllez, 2018).

Para Lucano (2013), el clima organizacional es un sistema de fundamental existencia dentro de las organizaciones, el cual brinda un direccionamiento a todos los empleados de la misma, ya sea compartiendo significados, valores, creencias, ideas e información. Establece también, la forma como los empleados piensan y actúan dentro de su puesto de trabajo, generando un clima de confianza, cooperación y ayuda mutua.

De acuerdo con Beltrán (2017), la calidad de vida del personal es pieza fundamental en la gestión del cambio, para el desarrollo del sector lechero. En estos procesos de cambio, se deben dar importancia al componente humano de las unidades productivas, lo cual repercutirá en el elevado rendimiento individual y colectivo del grupo de trabajo, todo en función del mejoramiento continuo.

Un buen clima organizacional ayuda y alienta a los trabajadores a ser competentes entre ellos, creando una conducta madura de todos los miembros, permitiendo que ellos se comprometan a ser responsables de sus asignaciones laborales dentro de la empresa (Calvache, 2014). La existencia de un grato ambiente laboral generará buenas relaciones laborales dentro de la organización, conllevando a trabajadores integrados, facilitando la cooperación entre todos (Carazas, 2017).

Asimismo, Quispe y Carlos (2014) resaltan la importancia de tener un buen clima laboral para lograr que el personal se encuentre altamente motivado, comprometido y productivo, a su vez esto se traduce a un mejor desempeño y óptima satisfacción laboral. Por otro lado, los comportamientos y consecuencias adversos a un clima organizacional armonioso que se presentan en una empresa lechera son la presencia de pensamientos cerrados, rechazo de las opiniones y sugerencias de los compañeros, generando discordancias y una mentalidad negativa de mejora.

Según lo mencionado anteriormente, resulta importante realizar el análisis del clima organizacional en empresas lecheras. En el Perú, por ejemplo, Quispe y Carlos (2014) realizaron el diagnóstico del clima organizacional de una empresa del sector agropecuario, dando a conocer que los recursos humanos no son considerados por la empresa de manera adecuada, existe poca motivación, las actividades son realizadas con desconfianza porque el entorno laboral es tenso, no se cuenta un plan de capacitación, malas relaciones entre trabajadores, chismes, incomodidades por condiciones laborales, y muchas otras situaciones que demuestran la poca identificación de los trabajadores con la empresa.

Se puede inferir que el talento humano es el capital más importante para el cumplimiento de cada uno de los objetivos de la empresa, ya que le dan un valor agregado a los productos y servicios (Quispe y Carlos (2014), por lo que se debe implementar un buen clima organizacional, caso contrario puede llegar a afectar la productividad. Por lo tanto, el clima organizacional tiene una repercusión directa en el bienestar de los empleados y debe ser tomado en cuenta en las diferentes empresas.

La presente investigación se realizó en una empresa lechera, dedicada a la comercialización de leche fresca en la provincia de Huaura, Perú. Esta empresa constituida desde el año 1982 se posiciona dentro de los mejores establos en el ranking de productividad de la cuenca lechera de Lima, por lo que se convierte en una empresa referente. Por ello, la presente investigación tiene como objetivo identificar la relación que existe entre el clima organizacional y el 
desempeño laboral de los colaboradores de esta empresa mediante un análisis correlacional. Con este fin, se aplicó un cuestionario a 40 colaboradores de las diferentes áreas de la empresa.

\section{DESARROLLO}

\section{Conceptualización de clima organizacional}

$\mathrm{El}$ ambiente existente entre los miembros de una organización se denomina "clima organizacional" o "clima laboral", el cual está relacionado con el grado de motivación de las personas. Es decir, se basa en los estados emocionales de los trabajadores. A su vez, estos estados son el resultado de la forma en como ellos perciben diferentes aspectos dentro de su empresa, por ejemplo, la comunicación con sus compañeros, el liderazgo de los jefes, sus relaciones interpersonales, la motivación que reciben, etc. (Chiavenato, 2011).

Para Osorio (2013), el clima organizacional interviene en los factores del sistema organizacional y las tendencias motivacionales, esto trae resultados sobre la productividad de la organización.

Asimismo, Gonçalves (2018) afirma que el comportamiento de un trabajador no es resultado de los factores organizacionales, sino de cómo el trabajador percibe estos factores. Estas percepciones dependen en buena medida de las actividades, interacciones y otra serie de experiencias que cada miembro tenga con la empresa. Los factores y la estructura del sistema organizacional generan a un determinado clima. Este clima resultante se ve reflejado en determinados comportamientos en los trabajadores. Estos comportamientos tienen un efecto en la organización y, por ende, en el clima organizacional, completando el circuito (ver figura 1).

\section{Figura 1}

Circuito de Interacciones del Clima Organizacional

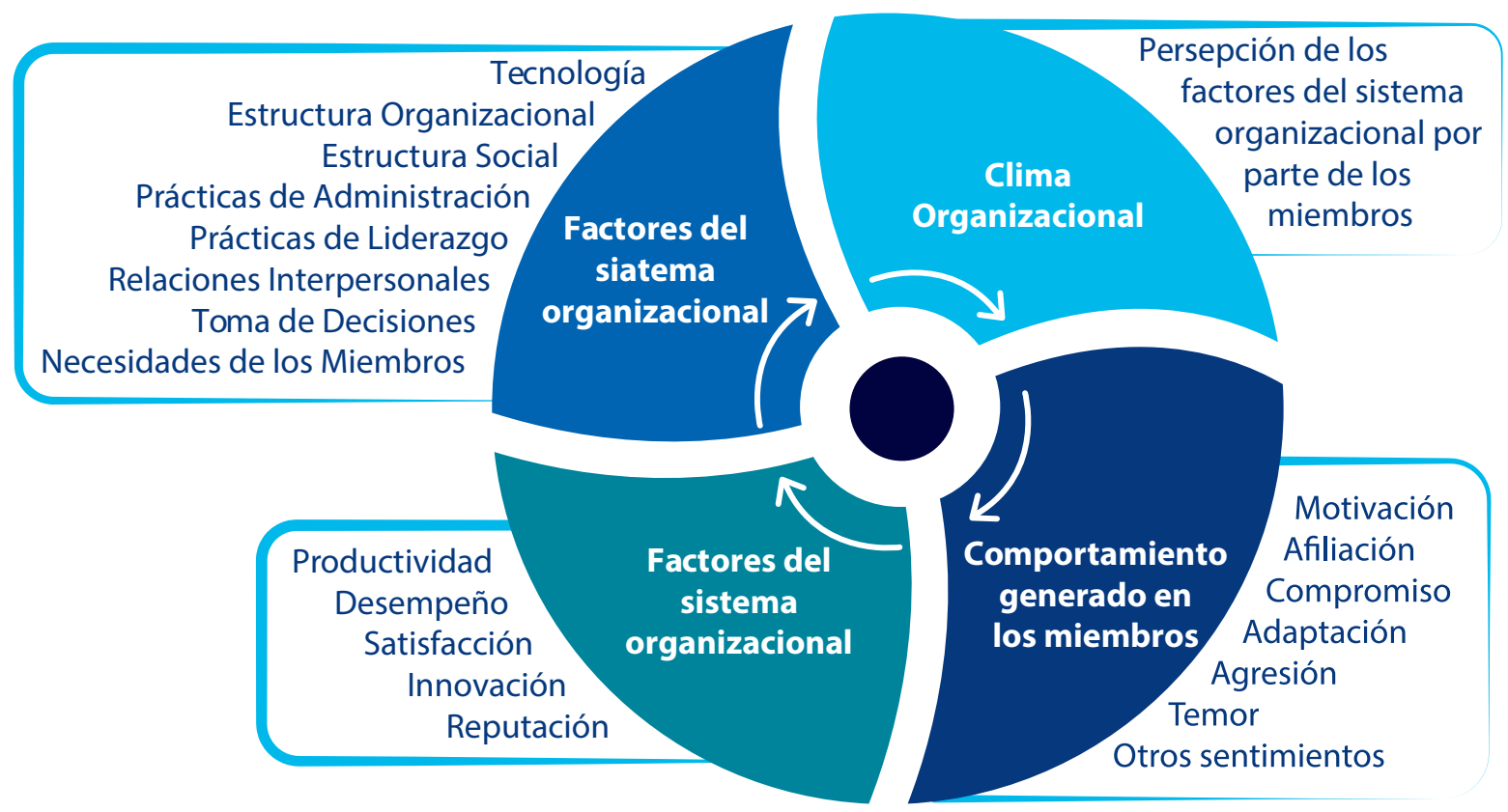

Fuente: elaboración propia. 
A partir de este esquema, podemos entender la importancia de generar un buen clima organizacional porque permite incrementar la productividad, disminuir el ausentismo, reducir costos y aumentar el desempeño que permita alcanzar el éxito (Salguero y García, 2017). Si bien, el clima organizacional busca ofrecer calidad de vida al trabajador, el administrador o encargado de una empresa debe saber identificar de donde nacen los conflictos, problemas e insatisfacciones que ocasionan que el trabajador tenga actitudes negativas hacia la empresa; es decir, identificar el clima que prevalece en la empresa que tiene a cargo (Córdova, 2017).

\section{Conceptualización de desempeño laboral}

El desempeño define el rendimiento laboral, es decir, la capacidad de una persona para producir, hacer, elaborar, acabar y generar trabajo en menos tiempo, con menor esfuerzo y mejor calidad, estando dirigido a la evaluación la cual dará como resultado su desenvolvimiento. El desempeño laboral es la eficacia del personal que trabaja dentro de las organizaciones, funcionando la persona con una gran labor y satisfacción laboral (Chiavenato, 2002, citado en Bonifacio y Falconi, 2016).

Para Chiavenato (2000), la evaluación del desempeño constituye una técnica de dirección imprescindible en la actividad administrativa. Es un medio que permite localizar problemas de supervisión de personal, integración del empleado a la organización o al cargo que ocupa en la actualidad, desacuerdos, desaprovechamiento de empleados con potencial más elevado al requerido por el cargo, motivación, etc.

Por otro lado, Quispe (2015) señala que, la productividad, eficacia y eficiencia laboral son dimensiones del desempeño laboral.

\section{METODOLOGÍA}

Para el desarrollo de esta investigación, se inició con la revisión bibliográfica para identificar las dimensiones del clima organizacional: liderazgo, relaciones interpersonales y motivación. La metodología que se utilizó es de tipo descriptiva, transversal y correlacional, porque se quiere conocer la influencia que tiene el clima organizacional sobre el desempeño laboral. La muestra estuvo conformada por 40 trabajadores de la empresa Inversiones Pecuarias Granados S.A.C, a quienes se le realizó un cuestionario de 17 preguntas abarcando las 3 dimensiones del clima organizacional, además de un cuestionario de 15 preguntas para la variable desempeño laboral (Tabla 1).

Tabla 1

Dimensiones de las Variables Utilizadas para el Cuestionario

\begin{tabular}{ccc}
\hline $\begin{array}{c}\text { Definición } \\
\text { operacional }\end{array}$ & Dimensiones & $\begin{array}{c}\mathbf{N}^{\circ} \text { de } \\
\text { preguntas }\end{array}$ \\
\hline $\begin{array}{c}\text { Clima } \\
\text { Organizacional }\end{array}$ & $\begin{array}{c}\text { Relaciones } \\
\text { Interpersonales } \\
\text { Motivación }\end{array}$ & $\mathbf{5}$ \\
\hline \multirow{2}{*}{$\begin{array}{c}\text { Desempeño } \\
\text { Laboral }\end{array}$} & Productividad & $\mathbf{6}$ \\
\hline & Satisfacción laboral & $\mathbf{5}$ \\
\hline
\end{tabular}

Fuente: elaboración propia. 
Para el análisis de correlación entre estas dos variables, se halló el coeficiente de correlación de Spearman, que es una medida para calcular la correlación (la asociación o interdependencia) entre dos variables aleatorias continuas. Para el cálculo de estos valores, se empleó el procesador Statistical Package of Social Sciencies (SPSS versión 24), versión en español, con ayuda del EXCEL 2010 para la tabulación de los datos. Además, se elaboró una tabla de doble entrada para una interpretación comprensible.

\section{RESULTADOS}

Se analizó de manera individual el Clima organizacional y el desempeño laboral, así como cada una de sus dimensiones y se generaron gráficas de los resultados para una mejor observación de resultados. A continuación, se muestran los resultados obtenidos de la encuesta aplicada a 40 trabajadores que laboran en la empresa Inversiones Pecuarias Granados S.A.C.

Figura 2

Respuesta Sobre el Clima Organizacional

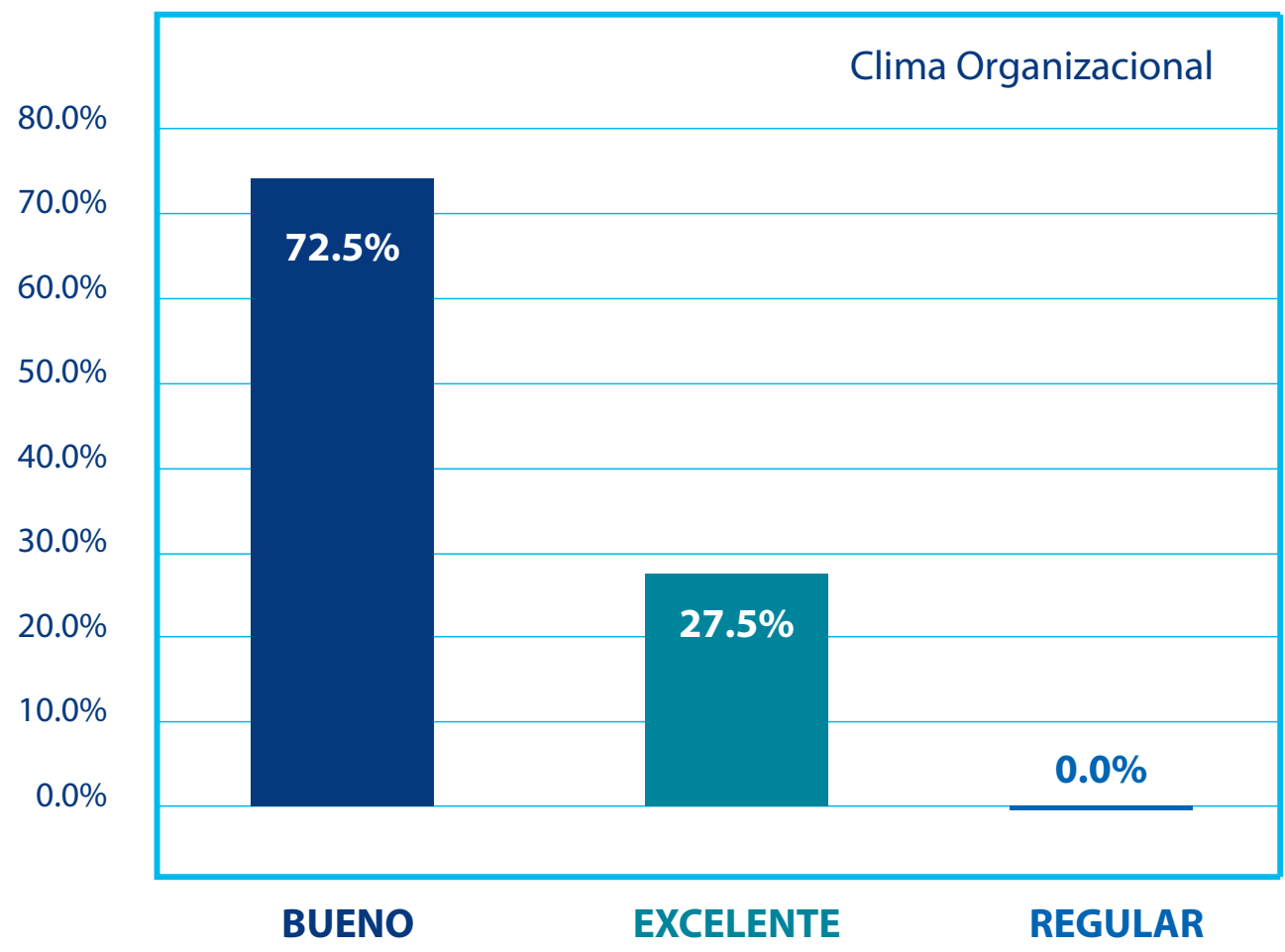

Fuente: elaboración propia. 
Para la variable Clima organizacional, en la Figura 2 se observa que según las encuestas realizadas, el $72.5 \%$ de los trabajadores expresan que existe buen clima organizacional, mientras que un $27.5 \%$ perciben la existencia de un excelente clima organizacional. Esto se reafirma con las dimensiones; para el liderazgo, el $97.5 \%$ de trabajadores indican que su jefe cuenta con aptitudes necesarias, capacidad para resolver conflictos, fomenta la mejora continua en la tecnología y los incentiva. Asimismo, el 97.5\% afirma que existen buenas relaciones interpersonales entre trabajadores. Por otro lado, $67.5 \%$ manifiesta que la motivación es buena porque la empresa reconoce y valora sus esfuerzos brindándoles beneficios económicos.

Para la variable Desempeño laboral, en la Figura 3 se observa que según las encuestas realizadas, el $82.5 \%$ de los trabajadores demuestran un excelente desempeño laboral y un $17.5 \%$ un buen desempeño. Esto es reflejo de las dimensiones evaluadas para esta variable; así para la productividad se registró un $92.5 \%$ de trabajadores con excelente productividad, un $80 \%$ con excelente competencia y habilidades, y un $57.5 \%$ percibe una buena satisfacción laboral.

Por otro lado, se obtuvieron los valores para el coeficiente de correlación de Spearman (Rho de Spearman) para evaluar el grado de relación existente entre la variable Clima organizacional y cada una de sus dimensiones con la variable Desempeño laboral. (Tabla 2).

Figura 3

Respuesta Sobre el Desempeño Laboral

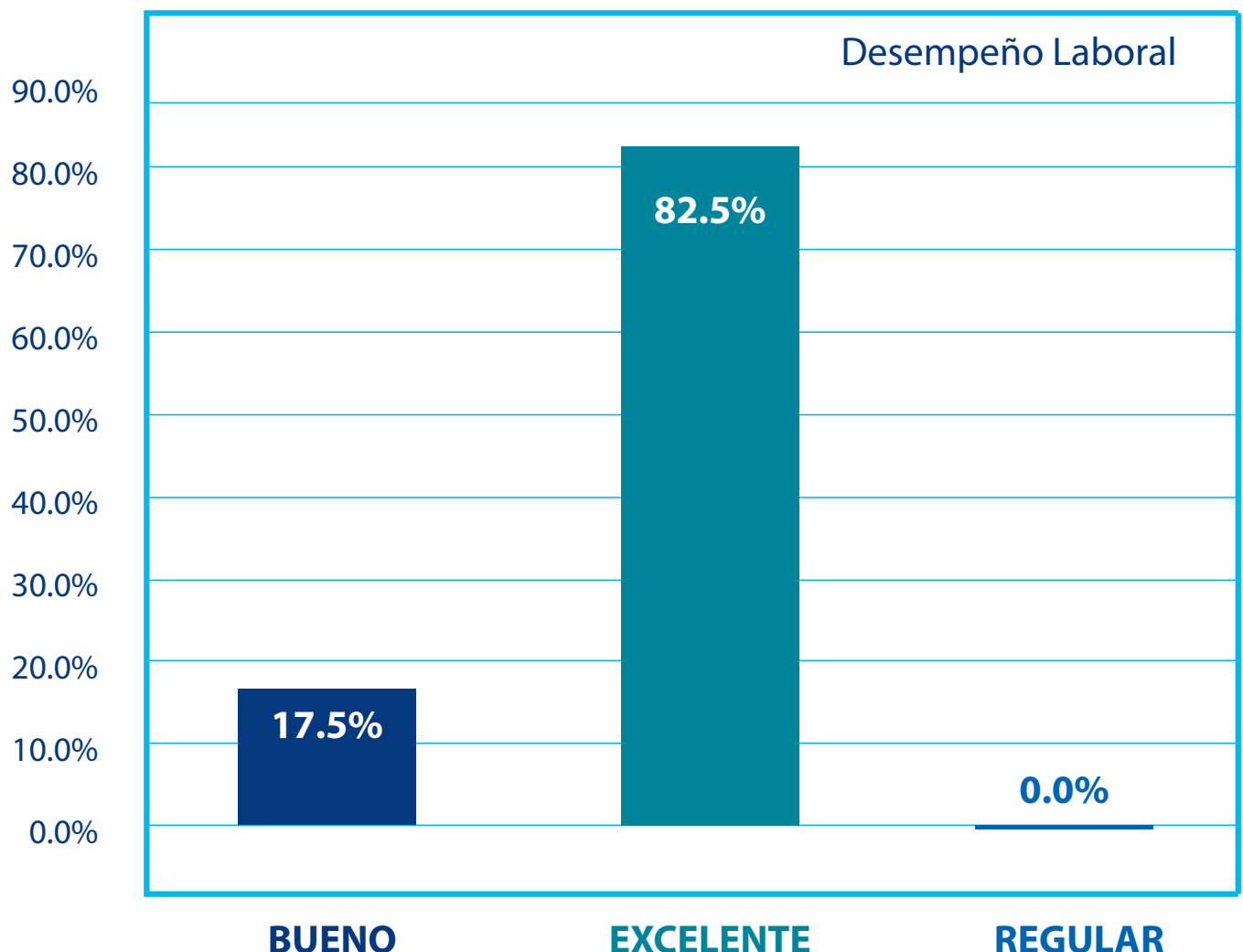

Fuente: elaboración propia. 
Los coeficientes de correlación demuestran que existe una relación moderada, positiva y significativa entre las diferentes dimensiones del clima organizacional y desempeño laboral; es decir, el clima organizacional tiene una influencia positiva sobre el desempeño laboral de los trabajadores en la empresa Inversiones Pecuarias Granados S.A.C. De forma similar, Jaya (2018) obtuvo una correlación positiva de 0.942 entre el clima organizacional y el desempeño laboral, confirmando que existe una relación directa entre estas dos variables.

\section{Tabla 2}

Rho de Spearman para Clima Organizacional y Desempeño Laboral.

\section{Variables}

Desempeño laboral

\begin{tabular}{ll} 
Liderazgo & $\mathbf{0 . 3 7 3 * *}$ \\
\hline Relaciones interpersonales & $\mathbf{0 . 5 2 3 * *}$ \\
\hline Motivación & $\mathbf{0 . 1 8 3}$ \\
\hline Clima Organizacional & $\mathbf{0 . 5 1 1 * *}$ \\
\hline
\end{tabular}

$\left.{ }^{* *}\right)$ La correlación es significativa 
En la presente investigación, el liderazgo tiene influencia positiva sobre el desempeño laboral, Bonifacio y Falconi (2016) indican que, si bien la relación entre estas variables es de grado bajo, el liderazgo tiene influencia positiva sobre el desempeño laboral. De acuerdo con Calvache (2014), el desempeño laboral de los trabajadores se ve afectado en gran parte por la aplicación del liderazgo autoritario porque impide que aporten nuevas ideas y en cierto los cohíbe, afectando el desarrollo del clima organizacional.

Para la empresa evaluada, la dimensión de las relaciones interpersonales, tiene una correlación que indica influencia positiva sobre el desempeño laboral de los trabajadores. Por ello, la calidad de vida laboral del personal, debe ser considerada como un componente fundamental de la gestión del cambio para el desarrollo del sector, lo cual repercutirá en el elevado rendimiento individual y colectivo del grupo humano de trabajo, todo en función del mejoramiento continuo (Beltrán, 2017). Es necesario monitorear las relaciones interpersonales entre los trabajadores, ya que uno de los principales problemas de toda empresa, es que muchas veces, una vez realizada la selección del personal, no hay un posterior seguimiento de cómo es que los nuevos trabajadores se acoplan con sus compañeros y cómo interactúa con las demás áreas de la empresa (Gamez, 2015).

En cuanto a los pensamientos cerrados, rechazo de las opiniones y sugerencias de los compañeros, generando discordancias y una mentalidad negativa de mejora, son considerados como comportamientos y consecuencias adversas a un clima laboral armonioso. Se sugiere que la existencia de un grato ambiente laboral generará buenas relaciones laborales dentro de la organización, conllevando a trabajadores integrados, facilitando la cooperación entre todos. (Carazas, 2017).

La dimensión de motivación obtuvo una correlación baja, sin embargo; esto sugiere que la motivación tiene influencia positiva sobre el desempeño laboral y si la empresa desarrolla factores que implican motivación positiva, se generará un comportamiento positivo en el desempeño de los trabajadores.

\section{CONCLUSIONES}

Después de realizar el análisis de los resultados obtenidos en esta investigación, se concluye que la empresa cuenta con un Clima organizacional favorable que influye positivamente en el desempeño de sus trabajadores. Además, con base en los resultados es necesario promover un programa de mejora continua del clima organizacional, incluyendo actividades extras como capacitaciones y talleres que permitan a la empresa encaminar mejores resultados y con ello un mejor desempeño laboral de sus trabajadores, lo cual se traduce en el logro de sus objetivos.

Por otra parte, los líderes de cada jefatura deben tomar en cuenta la opinión de los trabajadores con el fin de generar nuevas ideas lo cual les permitirá tener plena confianza en sí mismos, de manera tal que se beneficie el desarrollo y crecimiento de la empresa.

También, es necesario desarrollar actividades recreacionales y capacitaciones a todo el personal de la empresa, con el propósito de mejorar la comunicación y las relaciones entre trabajadores y optimizar el desempeño laboral de cada uno con miras a conseguir las metas trazadas por la empresa.

Implementar charlas motivacionales a todos los trabajadores con la finalidad de elevar la autorrealización, y del mismo modo, implementar escala de incentivos para mejorar la motivación de los trabajadores más destacados, lo cual los impulsará a conseguir los logros esperados para el nivel de productividad de la empresa. 


\section{REFERENCIAS}

Bonifacio, W. y Falconi, M. (2016). Clima organizacional y desempeño laboral de los empleados en la empresa sociedad agricola Virú S.A - Año 2016. Universidad Inca Garcilaso de la Vega, Chincha, Perú.

Beltrán, D. (2017). Caracterizacón del clima organizacional de las empresas tecnificadas de ganadería de leche de la provincia del Tundama en el departamento de Boyacá. Universidad Nacional de Colombia, Bogotá, Colombia.

Beltrán, D. y Téllez, G. (2018). Estudio de percepción del clima organizacional de las empresas tecni $\square$ cadas de ganadería de leche de la provincia del Tundama, Boyacá, Colombia. Revista de la Facultad de Medicina Veterinaria y de Zootecnia, $65(1)$.

Calvache, J. (2014). El clima organizacional y su incidencia en el trabajo en equipo de la empresa de productos lácteos el paraíso de la ciudad de Salcedo. Universidad Técnica de Ambato, Ecuador.

Carazas, N. (2017). Valoración y percepción de roles legitimados en la cotidianidad del trabajo y competitividad laboral, de los trabajadores de la empresa Laive S.A. Planta Arequipa - 2017. Universidad Nacional de San Agustín, Arequipa, Perú.

Chiavenato, I. (2000). Introducción a la Teoría General de la Administración. Editorial McGrawHill, México.

Chiavenato, I. (2011). Administración de Recursos Humanos: El Capital Humano de las Organizaciones. Editorial McGrawHill, México.

Córdova, D. F. (2017). Clima organizacional y satisfacción laboral de los asesores del centro mejor atención al ciudadano, Callao 2016. Universidad César Vallejo, Perú.

Gamez, R. (2015). Cultura y clima organizacional en empresas agrícolas. Revista Administracion \& Finanzas, 2(2), 159-169.

Gonçalves, A. (19 de Julio del 2018). Dimensiones del Clima Organizacional. Recuperado de http://www.geocities.ws/ janethqr/liderazgo/130.html

Jaya, S. (2018). Clima organizacional y su incidencia en el desempeño laboral en las Pymes del sector lácteo de la Parroquia Belisario Quevedo. Universidad Técnica de Cotopaxi, Latacunga, Ecuador.

Lucano, I. (2013). Clima Organizacional y valores en la empresa Gloria S.A.-La Libertad. Universidad Nacional de Trujillo, Perú.

Ministerio de Agricultura. (2017). Diagnóstico de crianzas priorizadas para el Plan Ganadero 2017-2021.

Osorio, J. R. (2013). Influencia del clima organizacional en el desempeño laboral del personal de la sede de la dirección regional de agricultura Junín. Universidad Nacional del Centro del Perú, Huancayo, Perú.

Quispe, H. y Carlos, J. (2015). Diagnóstico del clima organizacional, para incrementar el nivel de satisfacción de los trabajadores de la empresa Agropecuaria Chimu SRL, Trujillo - 2014. Universidad Privada Antenor Orrego, Trujillo, Perú.

Quispe, E. (2015). Clima organizacional y el desempeño laboral en la Municipalidad distrital de Pacucha, Andahuaylas, 2015. Universidad Nacional José María Arguedas, Andahuaylas, Perú.

Salguero, N. y García, C. (2017). Influencia del liderazgo sobre el clima organizacional en las instituciones de educación superior. Boletin virtual, (6-4), 135-149. 